\title{
Isolation and Characterization of Shiga Toxin-Producing Escherichia coli O157:H7 from Beef, Pork and Cattle Fecal Samples in Changchun, China
}

\author{
Zhijiang ZHOU ${ }^{1)}$, Yoshikazu NISHIKAWA ${ }^{2)}$, Ping ZHU ${ }^{1)}$, Shangyuan $\mathrm{HONG}^{4)}$, Atsushi HASE'), Tom CHEASTY ${ }^{3)}$, \\ Henry R. SMITH ${ }^{3)}$, Minggong ZHENG ${ }^{1)}$ and Kosuke HARUKI ${ }^{2)}$ \\ ${ }^{1)}$ Faculty of Animal Medicine, Changchun University of Agriculture and Animal Sciences, Changchun 130062, China, ${ }^{2)}$ Osaka City \\ Institute of Public Health and Environmental Sciences, Osaka 543-0026, Japan, ${ }^{3)}$ Laboratory of Enteric Pathogens, Central Public \\ Health Laboratory, London NW9 5HT, United Kingdom and ${ }^{4)}$ Department of Microbiology, 302 Hospital, Beijing 100039, China
}

(Received 7 March 2001/Accepted 8 July 2002)

ABSTRACT. Meat samples and fecal specimens from adult cattle were collected in Changchun, China and were examined for presence of Shiga toxin-producing Escherichia coli (STEC) serogroup O157. STEC O157 strains were isolated from 2 (5\%) of 40 beef, 1 (3.3\%) of 30 pork, and $3(1.7 \%)$ of 176 adult cattle fecal samples. The strains belonged to phage types (PT) 4, 8, or 47 . Two beef strains and a strain previously isolated from a patient in Shandong, China, were PT-4 and showed a similar PFGE pattern, suggesting the poss ibility of food-borne transmission. It is suggested that cattle are a reservoir of STEC O157:H7 and meat products are contaminated by this pathogen in Changchun, China as well as in other countries. KEY WORDS: China, Escherichia coli, O157.

J.Vet.Med. Sci. 64(11): 1041-1044, 2002

Shiga toxin (Stx)-producing Escherichia coli (STEC) serogroup O157 (O157:H7 or O157:NM [non-motile]) has been associated with a variety of human diseases, including mild diarrhea, hemorrhagic colitis, and diarrhea-associated form of the HUS since it was first described in 1982 [6]. Humans become infected with STEC O157 following the consumption of contaminated foods or water or by direct transmission from infected humans or animals $[1,3,6]$. Undercooked ground beef and raw milk have most often been implicated in food-borne infections. Since healthy domestic animals, in particular, ruminants like cattle, sheep, and goats can harbor STEC O157 in their feces, they have been regarded as natural reservoirs of this pathogens [2]. In China, outbreaks due to STEC O157 have not been reported. Although several sporadic cases of STEC O157 infection have been reported $[10,12,17,18]$, the sources of in these cases have not been defined. To assess the significance of meat and cattle as the vehicle or the reservoir, beef, pork and cattle fecal samples were collected in the Changchun area and examined for STEC O157. The isolates were characterized to assess their diarrheagenicity and were analyzed for epidemiological comparison.

In the period from September to November 1996, beef and pork samples were collected at several free markets of Changchun. The samples were brought to the laboratory and examined for STEC O157 within $1 \mathrm{hr}$. Fecal samples from adult cattle were collected at two farms and two slaughterhouses of Changchun between May and August 1997. The swabs very heavily coated with feces were transported immediately to laboratory at ambient temperature. Upon arrival at the laboratory, the swabs were analyzed either immediately or held at $4^{\circ} \mathrm{C}$ no longer than $48 \mathrm{hr}$ before analysis.

A $25 \mathrm{~g}$ of portion of each beef and pork sample was aseptically removed and added to $225 \mathrm{ml}$ of modified trypticase soy broth (mTSB) in a 500-m $l$ flask. The mTSB enrichment medium was composed of (per liter) Trypticase soy broth (TSB;30 g; Sigma Chemical Co., St. Louis, Mo., U.S.A.), bile salt 3 (1.5 g; Difco Laboratories, Detroit, Mich., U.S.A.), dipotassium phosphate (1.5 g), and novobiocin (20 mg; Sigma Chemical Co., St. Louis, Mo., U.S.A.). The mTSB enrichment medium was prepared by the method previously described [5]. For cattle fecal samples, each sample swab was added to $225 \mathrm{~m} l$ of $\mathrm{mTSB}$ in a $500-\mathrm{m} l$ flask. The samples were incubated for 18 to $24 \mathrm{hr}$ at $37^{\circ} \mathrm{C}$. Each enrichment culture was spread plated onto sorbitol MaConkey agar (SMAC) [13]. SMAC medium was prepared as follows with MacConkey agar base (Difco Laboratories, Detroit, Mich., U.S.A.), which is identical to MacConkey agar but contains no lactose. MacConkey agar base (40.0 g) and D-sorbitol (10.0 g) (Difco) were mixed in 1 liter of distilled water, autoclaved, and poured into plates for use. The SMAC plates were incubated at $37^{\circ} \mathrm{C}$ for $24 \mathrm{hr}$. Up to 10 non-sorbitol fermenting (NSF) colonies were selected from each SMAC plate and streaked onto eosin methylene blue agar (EMB) (Difco Laboratories, Detroit, Mich., U.S.A.). The EMB plates were incubated at $37^{\circ} \mathrm{C}$ overnight. Those isolates with a typical $E$. coli metallic sheen on EMB were identified by biochemical tests. The isolates were confirmed to be of serotype O157:H7 or serotype O157:NM with an E. coli O157 latex test kit (Oxoid Ltd., Basingstoke, Hampshire, UK) and E. coli $\mathrm{O}$ - and $\mathrm{H}$-specific antisera (Denka Seiken Co., Ltd., Tokyo, Japan).

The presence of stx1, stx2, eaeA, eae O157, or fliC $h 7$ was examined by PCR as described previously [7, 8, 15]. Table 1 describes oligonucleotide primers used in the PCR reaction mixture. The primers used to detect the presence of stx2, stx2vha, stx2vhb gene sequences are also listed in Table 1. Stx 2 genotype of $E$. coli isolates were determined according to the method of Tyler et al. [16]. In brief, $10 \mu l$ 
Table 1. Oligonucleotide primers used in the study

\begin{tabular}{|c|c|c|c|c|}
\hline Primers & Oligonucleotide sequence $\left(5^{\prime}-3^{\prime}\right)$ & Product size (bp) & Target genes & Reference \\
\hline VT1a & GAAGAGTCCGTGGGATTACG & 130 & stxl & 15 \\
\hline VT1b & AGCGATGCAGCTATTAATAA & & & \\
\hline VT2a & TTAACCACACCCACGGCAGT & 346 & $s t x 2$ & 15 \\
\hline VT2b & GCTCTGGATGCATCTCTGGT & & & \\
\hline AE9 & ACGTTGCAGCATGGGTAACTC & 815 & eaeA & 7 \\
\hline AE10 & GTCCACTTTGACAACGGCTAG & & & \\
\hline AE19 & CAGGTCGTCGTGTCTGCTAAA & 1087 & eaeO157 & 8 \\
\hline AE20 & TCAGCGTGGTTGGATCAACCT & & & \\
\hline FLICH7-F & GCGCTGTCGAGTTCTATCGAGC & 625 & fliCh7 & 8 \\
\hline FLICH7-R & CAACGGTGACTTTATCGCCATTCC & & & \\
\hline VT2-c & AAGAAGATGTTTATGGCGGT & 285 & stx 2, stx $2 v h a$, stx $2 v h b$ & 16 \\
\hline VT2-d & CACGAATCAGGTTATGCCTC & & & \\
\hline VT2v-1 & CATTCACAGTAAAAGTGGCC & 385 & stx $2 v h a$, stx $2 v h b$ & 16 \\
\hline VT2v-2 & GGGTGCCTCCCGGTGAGTTC & & & \\
\hline
\end{tabular}

aliquots of amplification reaction mixture recovered after the PCR using the VT2-c and VT2-d primers were subjected to restriction endonuclease digestion with HaeIII, RsaI, or NciI (New England BioLab Inc., Beverly, MA, U.S.A.) as recommended by the manufacturer. The restriction endonuclease digestion of this amplicon permitted prediction of specific stx 2 , st $x 2 v h a$ and $s t x 2 v h b$ on the basis of predetermined restriction fragment length polymorphisms.

Isolates were grown overnight at $37^{\circ} \mathrm{C}$ with shaking (120 $\mathrm{rpm}$ ) in $5 \mathrm{ml}$ Casamino acid-yeast extract medium (Difco Laboratories, Detroit, Mich., U.S.A.). A $0.5 \mathrm{ml}$ portion of each culture was transferred into an Eppendorf tube and 25 $\mu l$ of 5,000 U polymyxin B was added. After incubation at $37^{\circ} \mathrm{C}$ for $30 \mathrm{~min}$, the supernatants were obtained by centrifuging the cultures at $15,000 \mathrm{rpm}$ for $2 \mathrm{~min}$. Stx production was determined by the reversed-passive latex agglutination with VTEC-RPLA kit (Denka Seiken) according to the manufacturer's instructions. Further, STEC O157 isolates were characterized by the pattern of adherence to HEp-2 cells as described previously [14].

Isolates confirmed as STEC O157 were phage typed at the Laboratory for Enteric Pathogens, Central Public Health Laboratory, London, UK by the scheme of Khakhria et al. [11]. As a molecular epidemiological method pulsed field gel electrophoresis (PFGE) was performed according to the method of Izumiya et al. [9]. XbaI (Takara Shuzo, Otsu, Shiga, Japan) was used as the restriction endonuclease. Digested DNAs were separated by electrophoresis through a $1 \%$ agarose gel by using a CHEF DR II apparatus (Bio-Rad, Hercules, CA, U.S.A.) in $0.5 \times$ Tris-borate-EDTA buffer at $14^{\circ} \mathrm{C}$ with a voltage of $200 \mathrm{~V}$. A linearly ramped switching time from 4 to $8 \mathrm{~s}$ was applied for $11 \mathrm{hr}$ and then a linearly ramped switching time from 8 to $50 \mathrm{~s}$ was applied for $9 \mathrm{hr}$.
In 1996, STEC O157 strains were isolated from $2(5 \%)$ of 40 beef samples and 1 (3.3\%) of 30 pork samples (Table 2). Food products have been the most frequently implicated source of STEC O157 in outbreaks and sporadic cases. Beef and other red meats have been considered to be highest risk foods. In an early study, STEC O157 was isolated from $3.7 \%$ of retail beef, $1.5 \%$ of pork samples in the United States and Canada [5]. This present study demonstrated that meat products were contaminated by this pathogen in a high percentage in the Changchun area. If the immunomagnetic separation method had been used, this rate would have been higher because this method increases the recovery of STEC O157 from the selective enrichment broth [4].

In 1997, STEC O157 strains were isolated from 3 (1.7\%) of 176 fecal samples from adult cattle (Table 2). In addition a strain of STEC O8 was isolated from one of these samples. Only the NSF isolates were examined in this study. These STEC strains were only isolated from 146 fecal samples of beef cattle at slaughterhouses, and no STEC strain was recovered from 30 fecal samples from cattle on farms. The origins of the animals and the origin of meat positive for STEC 0157 could not be traced. Reported estimates of the prevalence of STEC O157 in North America and European cattle range from 0 to almost $10 \%$ [1]. Our results suggest that cattle are a reservoir of STEC O157 in China as well as in other countries.

Properties of the STEC O157 strains are presented in Table 2. All STEC O157 isolates from cattle, beef and pork possessed stx and eae genes. The organisms produced Stx and adhered to HEp-2 cells in a localized manner which is characteristic of wild strains possessing LEE [14]. The strains were definitely potential human pathogens.

Three phage types (PT-4, 8 and 47) were identified 
Table 2. Characterization of STEC O157 isolates from beef, pork and fecal specimens

\begin{tabular}{|c|c|c|c|c|c|c|c|c|c|}
\hline \multirow[t]{2}{*}{ Isolates } & \multicolumn{4}{|c|}{ Virulent genes } & \multicolumn{2}{|c|}{ RPLA titer } & \multirow[t]{2}{*}{ Phage type } & \multirow{2}{*}{$\begin{array}{l}\text { Adherence to } \\
\text { HEp-2 cell }\end{array}$} & \multirow[t]{2}{*}{ Origin } \\
\hline & stxl & stx 2 & stx $2 v h a$ & eae & Stx 1 & Stx2 & & & \\
\hline 96001 & + & + & - & + & 80 & 160 & 4 & $\mathrm{LA}^{\mathrm{b})}$ & Beef \\
\hline 96005 & + & + & - & + & 80 & 160 & 4 & LA & Beef \\
\hline 96009 & - & + & + & + & - & 2560 & 47 & LA & Pork \\
\hline 97063 & + & + & - & + & 160 & 1 & $\mathrm{RDNC}^{\mathrm{a})}$ & LA & Cattle feces \\
\hline 97094 & + & + & + & + & 160 & 1 & 8 & LA & Cattle feces \\
\hline 97168 & + & + & + & + & 80 & 1 & 8 & LA & Cattle feces \\
\hline $94 \mathrm{H}$ & + & + & - & + & 640 & 320 & 4 & LA & Human feces \\
\hline
\end{tabular}

a) RDNC: this culture Reacts with typing phages but Does Not Conform to a recognized pattern.

b) LA: localized adherence.

among the 7 food, animal and human STEC O157 isolates; one of the STEC 0157 from cattle feces did not conform to a recognized pattern (Table 2). A strain of STEC O157 that was isolated from a patient in 1994 was compared with the other strains by PFGE. No identical PFGE pattern was recognized in the isolates tested, but a very similar pattern was seen with two beef isolates 96001, 96005 and a human isolate $94 \mathrm{H}$ (Fig. 1). They all belonged to PT-4, and PT-4 is one of the most common phage types of human isolates in North America[11]. The similar PFGE patterns and identical phage type between two beef isolates and one human isolate suggest that they might have a relationship. It is likely that meat products have played a role in the transmission of STEC O157 to human in China.

PT- 4 and 8 have been frequently isolated from both human and non-human sources in many other countries, but PT-47 was originally reported to be the type unique to Japan. Khakhria et al. [11] reported that some phage types were common worldwide, some were found only in definite geographical area. Types 49, 50, 51, and 52 were isolated in the U. K. but not in Canada. Within Canada, types 36, 38 and 53 were observed only in British Columbia and types 55 and 58 only in Alberta. To our knowledge, however, PT-47 has not been detected, even in Japan, after the report of Khakhria et al. [11]. We found it among only 7 isolates, so it might be a type restricted in East Asia area.

To our knowledge this is the first report of isolation of STEC 0157 from meat and cattle feces in China. It can be concluded that cattle are natural hosts of STEC O157 in China with beef and pork contaminated by this pathogen in the Changchun area. Further investigations should be performed throughout China to define the association between this pathogen in cattle and meat and as a cause of human disease.

ACKNOWLEDGMENTS. This project was made possible through research funding No. 39670563 from the National Natural Science Foundation of China, and partially supported by a grant of Osaka Fellowship of Osaka City, Japan.

\section{REFERENCES}

1. Armstrong, G.L., Hollingsworth, J. and Morris, J.G.Jr. 1996.

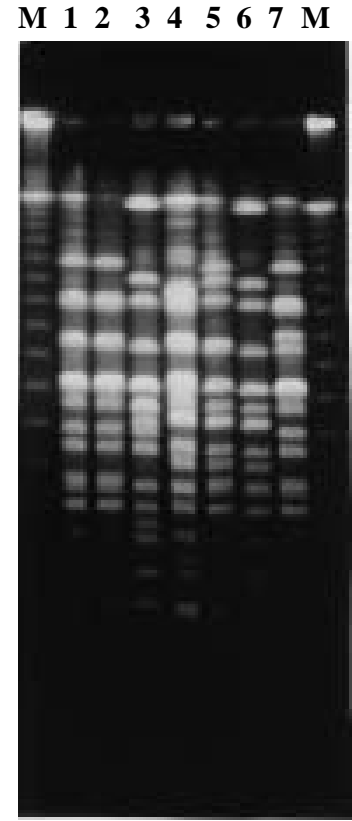

Fig. 1. PFGE of STEC O157:H7 strains isolated from Changchun, China. Lanes M, molecular weight markers (lambda ladder standard); 1, STEC O157:H7 96001; 2, STEC O157:H7 strain 96005; 3, STEC O157:NM strain 96009; 4, STEC O157:H7 97063; 5, STEC O157:H7 strain 97094; 6, STEC O157:H7 strain 97168; 7, STEC O157:H7 strain $94 \mathrm{H}$.

Epidemiol. Rev. 18: 29-51.

2. Beutin, L., Geier, D., Steinruck, H., Zimmermann, S. and Scheutz, F. 1993. J. Clin. Microbiol. 31: 2483-2488.

3. Boyce, T.G., Swerdlow, D.L. and Griffin, P.M. 1995. New. Engl. J. Med. 333: 364-368.

4. Chapman, P.A., Siddons, C.A., Zadik, P.M. and Jewes, L. 1991. J. Med. Microbiol. 35: 107-110.

5. Doyle, M.P. and Schoeni, J.L. 1987. Appl. Environ. Microbiol. 53: 2394-2396.

6. Griffin, P.M. and Tauxe, R.V. 1991. Epidemiol. Rev. 13: 6098.

7. Gannon, V.P.J., Rashed, M., King, R.K. and Thomas, E.J.G. 1993. J. Clin. Microbiol. 31: 1268-1274. 
8. Gannon, V.P.J., D’Souza, S., Graham, T., King, R.K., Rahn, K. and Read, S. 1997. J. Clin. Microbiol. 35: 656-662.

9. Izumiya, H., Terajima, J., Wada, A., Inagaki, Y., Itoh, K.I., Tamura, K. and Watanabe, H. 1997. J. Clin. Microbiol. 35: $1675-1680$.

10. Kain, K.C., Barteluk, R.L., Kelly, M.T., Xin, H., Hua, G.D., Yuan, G., Proctor, E.M., Byrne, S. and Stiver, H.G. 1991. J. Clin. Microbiol. 29: 90-95.

11. Khakhria, R., Duck, D. and Lior. H. 1990. Epidemiol. Infect. 105: 511-520.

12. Li, J., Zhou, G.Q., Wen, X.O., Cui, S.Y., Meng, W., Wang, Y.L. and Sun, Q.H. 1994. Chinese J. Zoonoses 10: 23-25. (In Chinese.)

13. March, S.B. and Ratnam, S. 1986. J. Clin. Microbiol. 23: 869-
872.

14. Nishikawa, Y., Scotland, S.M., Smith, H.R., Willshaw, G.A. and Rowe, B. 1995. Microbial. Pathogenesis 18: 223-229.

15. Pollard, D.R., Johnson, W.M., Lior, H., Tyler, S.D. and Rozee, K.R. 1990. J. Clin. Microbiol. 28: 540-545.

16. Tyler, S.D., Johnson, W.M., Lior, H., Wang, G. and Rozee, K.R. 1991. J. Clin. Microbiol. 29: 1339-1343.

17. Xu, J.G., Quan, T.S., Xiao, D.L., Fan, T.R., Li, L.M., Wang, C.A., Li, W. and Liu, H.M. 1990. Curr. Microbiol. 20: 299304.

18. Zhang, Z.G., Li, L., Zhang, B.A., Li, Y.F., Hong, Q.Y. and Feng, S.X. 1994. Chinese J. Med. Lab. Sci. 17: 235 (in Chinese). 